\title{
PICTORIAL REVIEW ON CONSTELLATION OF IMAGING FINDINGS OF BRAIN DEATH ON 3T MRI
}

\author{
Umamaheswara V. Reddy¹, Praveen Kumar Kolla², Amit Agrawal ${ }^{3}$, Kishor V. Hegde', \\ Rakesh Borrugada ${ }^{1}$, Priya Malpani ${ }^{1}$ \\ ${ }^{1}$ Department of Radiology, Narayana Medical College Hospital, Chinthareddypalem, Nellore, \\ Andhra Pradesh, India \\ ${ }^{2}$ Department of Nephrology, Narayana Medical College Hospital, Chinthareddypalem, Nellore, \\ Andhra Pradesh, India \\ ${ }^{3}$ Department of Neurosurgery, Narayana Medical College Hospital, Chinthareddypalem, Nellore, \\ Andhra Pradesh, India
}

\begin{abstract}
Brain death is physiological loss of function of all intracranial structures above foramen magnum. Imaging is ancillary and are not substitute for clinical diagnosis of brain death. MRI can be useful supplementary diagnostic test for diagnosing brain death especially in cases where bed side clinical tests cannot be performed. No single sign in MRI is specific for brain death. Tonsillar herniation, loss of flow void in bilateral internal carotid arteries and vertebral arteries, swollen cortical gyri, edematous, cerebellar foliae with white matter hyperintensities, effacement of the ventricular system, uncal herniation compressing over the midbrain, brain stem swelling, dilated veins showing increased susceptibility phenomenon due to increase deoxyhemoglobin, diffuse restriction in cerebral cortex are some of the feature of brain death we present the imaging signs brain which we came across in two patients.
\end{abstract}

Keywords: brain death, tonsillar herniation, brain stem reflexes, Imaging signs, 3Tesla MRI

\section{INTRODUCTION}

Brain death is physiological loss of function of all intracranial structures above foramen magnum. $(1,2)$ It is complete irreversible cessation of all brain functions. $(1,2)$ Criteria for diagnosing brain death vary from country to country and from region to region. $(2,3)$ Primarily brain death is a clinical diagnosis: coma from a known cause, absent brain stem reflexes and prolonged apnoeic spells (3-10 mins without ventilator support) are required clinical criteria for diagnosing brain death. $(2,3)$ Imaging findings are ancillary and are not substitute for clinical diagnosis. $(2,3)$ MRI can be an useful supplementary diagnostic test for diagnosing brain death especially in cases where bed side clinical tests cannot be performed. $(2,4)$ Unlike other imaging tests ominous signs of brain death in MRI can be found without administering any contrast agent. $(2,4)$ for In our case we describe non contrast imaging features of brain on 3T MRI death in form of a pictorial assay.

\section{CASE REPORT}

\section{Case 1}

A 37 year old hypertensive male patient with chronic renal failure on regular haemodialysis was brought to emergency department in a comatose state. Few minutes later patient had an episode of cardiac arrest from which he was revived. Patient

Author for correspondence:

Amit Agrawal (MCh) Professor, Department of neurosurgery Neurosurgery, Medical College Hospital Chinthareddypalem Nellore-524003,

Andhra Pradesh, India

E-mail: dramitagrawal@gmail.com 
was diagnosed to have sepsis based on clinical findings and laboratory investigations. Patient was intubated and ventilator support was instituted. There was no clinical improvement during the ventilator support patient continued to be in coma and brain stem reflexes were absent. Patient was clinically declared to be brain dead as he had prolonged apnea on disconnecting the ventilator. MRI was performed for confirmatory evidence of brain death.

\section{Case 2}

A 26 year old female primigravida, not booked for antenatal checkups, presented to our hospital in status epileptics. Patient was intubated and was provided ventilator support immediately after administering necessary anticonvulsive medications. Recorded blood pressure at admission was 240/136 $\mathrm{mm} / \mathrm{Hg}$. Seizure activity was controlled, however her blood pressure was persistently high. Termination of pregnancy was done in favour of health of the mother. On 2nd day, patient had one more episode of seizure. MRI was advised by neurologist to know the cause of the persistent seizure activity. However patient was presumed to be brain dead during the transit to MRI console and MR imaging features confirmed the same.

MRI was performed on 3T GE HDXT machine in both patients with $\mathrm{T} 1, \mathrm{~T} 2$ spin echo, diffusion weighted imaging and susceptibility weighted imaging, which showed constellation of features consistent with brain death:

Tonsillar herniation (Fig. 1), loss of flow void in bilateral internal carotid arteries and vertebral arteries (Fig. 2), swollen cortical gyri showing T2 hyperintensities (Fig. 3) oedematous cerebellar foliae (Fig. 4) effacement of ventricular system (Fig. 5), uncal herniation bilaterally causing obliteration of cisterns and compression over the brain stem (Fig. 6 ), increased susceptibility phenomenon due to increase in deoxyhemoglobin (Fig. 7), diffuse DWI restriction in cerebral cortex (Fig. 8).

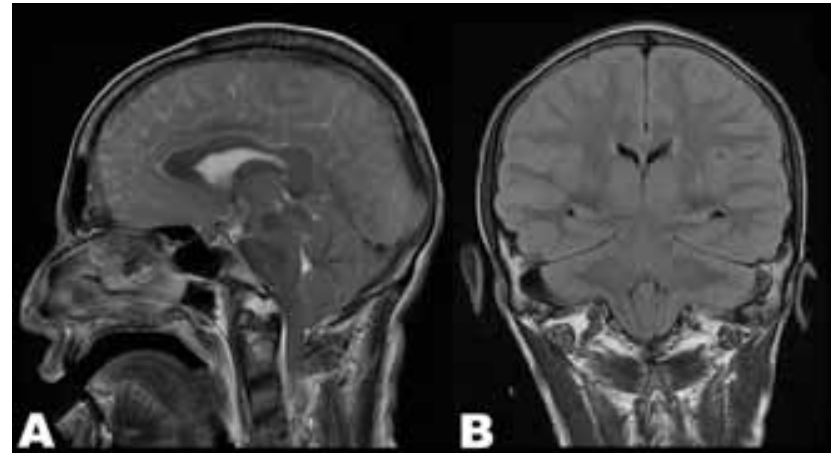

FIGURE 1. Sagittal T2W image (A) Coronal FLAIR image (B) showing herniation of the cerebellar tonsils below the foramen magnum. Hypothalamus, mammillary bodies, tegmentum are inferiorly displaced and obliterating the perimesencephalic cistern. Torcular herophili is pushed inferiorly.

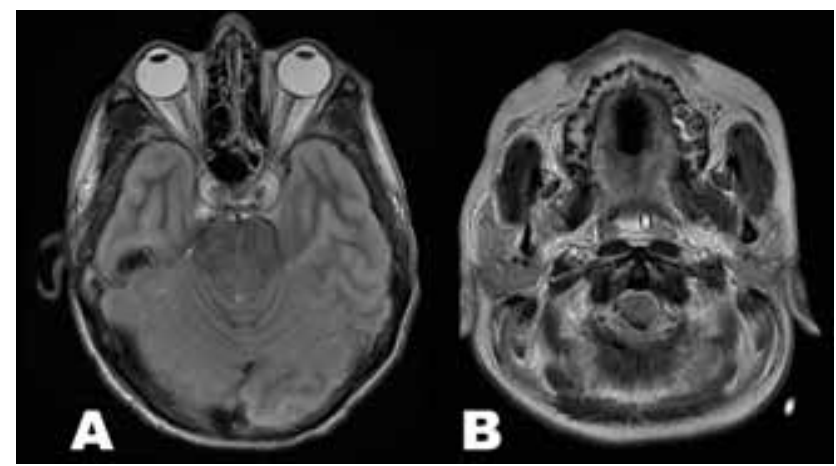

FIGURE 2. Axial, T2W image at the level of orbit (A) and just below level of foramen magnum (B) showing loss of flow void in intracranial, extracranial carotids and vertebral arteries.

\section{DISCUSSION}

MR imaging features consistent with brain death have been described earlier by many authors. $(2,4$, 5) According to Chul-Ho Sohn et al, most important MR imaging findings which are very sensitive and specific for brain death are loss of intracranial flow voids in carotids and vertebral arteries and tonsillar herniation. ${ }^{2}$ Stagnation of the blood with-
FIGURE 3. Axial T2W images $A, B, C, D$ showing swollen gyri and cortical T2 weighted hyperintensities

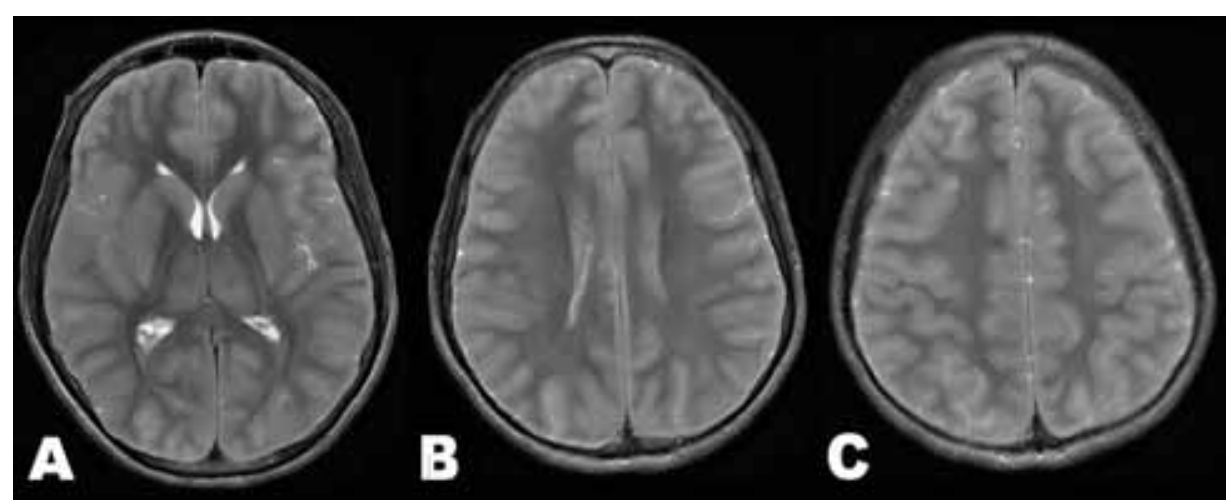




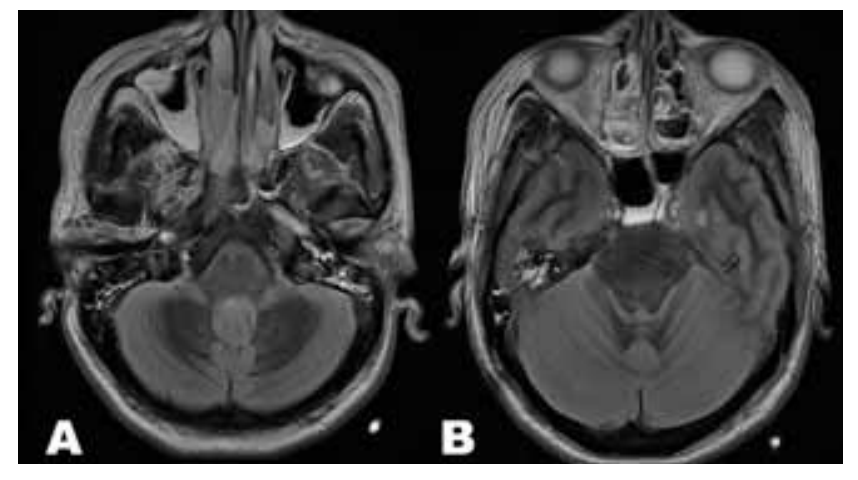

FIGURE 4. Axial T2W images at the level of cerebellum showing edema of the cerebellar foliae and effacement of the fourth ventricle, enlargement of the medulla and pons obliterating the basal cisterns. Flow voids in petrous portion of the carotids and pansinusitis were also seen.

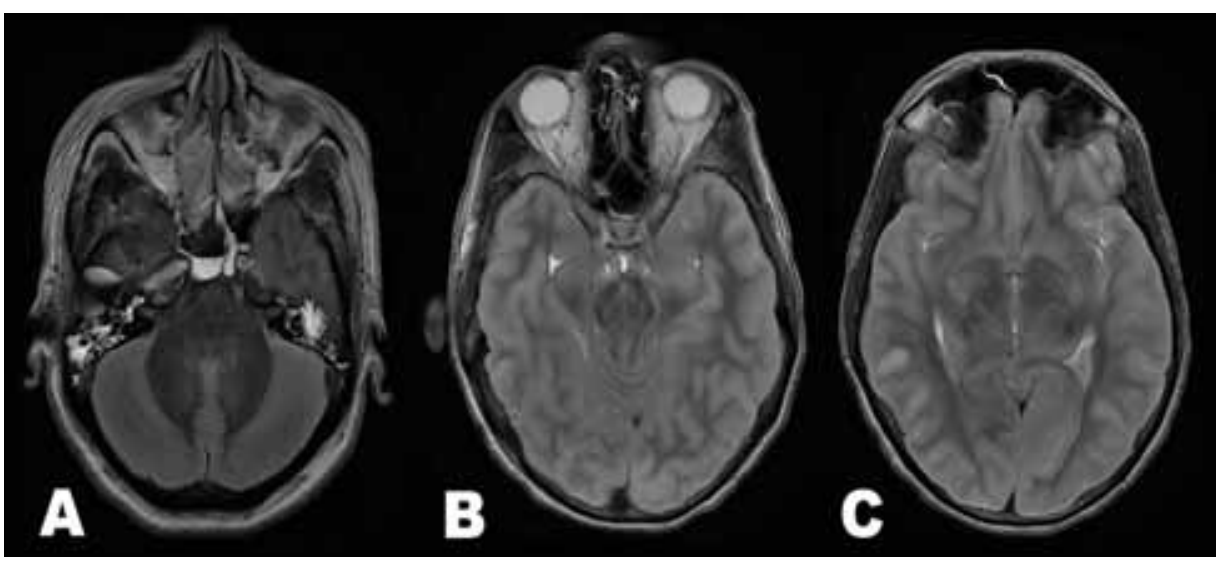

FIGURE 5. Axial T2W images $A, B, C$ showing effacement of the fourth ventricle, obliteration of the aqueduct, third ventricle and temporal horns of lateral ventricles.

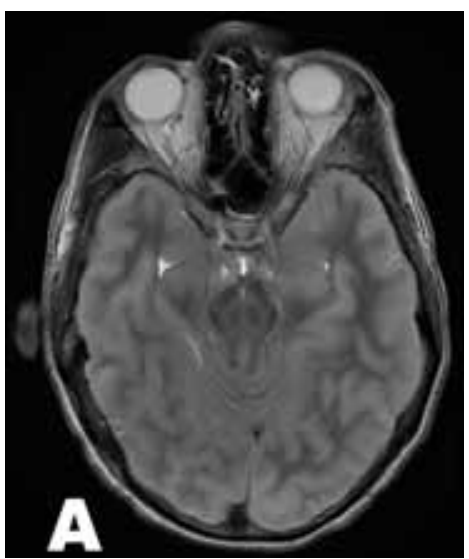

FIGURE 6. Axial T2W image at the level of the mid brain showing squeezing of the midbrain by herniated medial temporal lobes bilaterally.

in the carotid arteries due to increased intracranial pressure results in loss of flow void sign on T2. (5) Increased intracranial pressure and cortical swelling pushes the supratentorial structures down, inturn elevating the posterior fossa pressures and thus resulting in tonsillar herniation through foramen magnum. Diffuse gyral swelling conspicuous as T2W hyperintensities are consistently seen in brain death along with thalamic, caudate and cerebellar folial hyperintensites, however similar findings can be seen in profound hypoxia. $(2,5)$ Uniform diffusion restriction of supratentorial cortex can be seen in brain death, but even this finding is not specific as it can be found in many encephalopathies. $(2,4)$ The edematous pressure in supratentorial brain parenchyma causes descending tentorial herniation owing to compression of the mid brain by the uncus bilaterally. In severe cases there will be obliteration of the basal cisterns and squeezing of the mid brain. Intracranial pressure from supratentorial brain parenchyma also tends to buckle the optic chiasm, hypothalamus, mammillary bodies and mid brain against the base of skull. The basilar artery gets trapped in between enlarged pons and the clivus. Few other signs like effacement of the entire ventricular system have also been described on MRI. GRE/SWI findings in brain death are prominent transparenchymal and cortical veins which are a result of increase in deoxygenated blood and ischemia induced vasodilator release. (2) These findings are not specific to brain death and can be found in any ischemic event. (2) Diffusion weighted imaging shows homogenous diffusion restriction in the entire cortex and cerebellum consistent with cellular death. (4) None of the MRI features are 


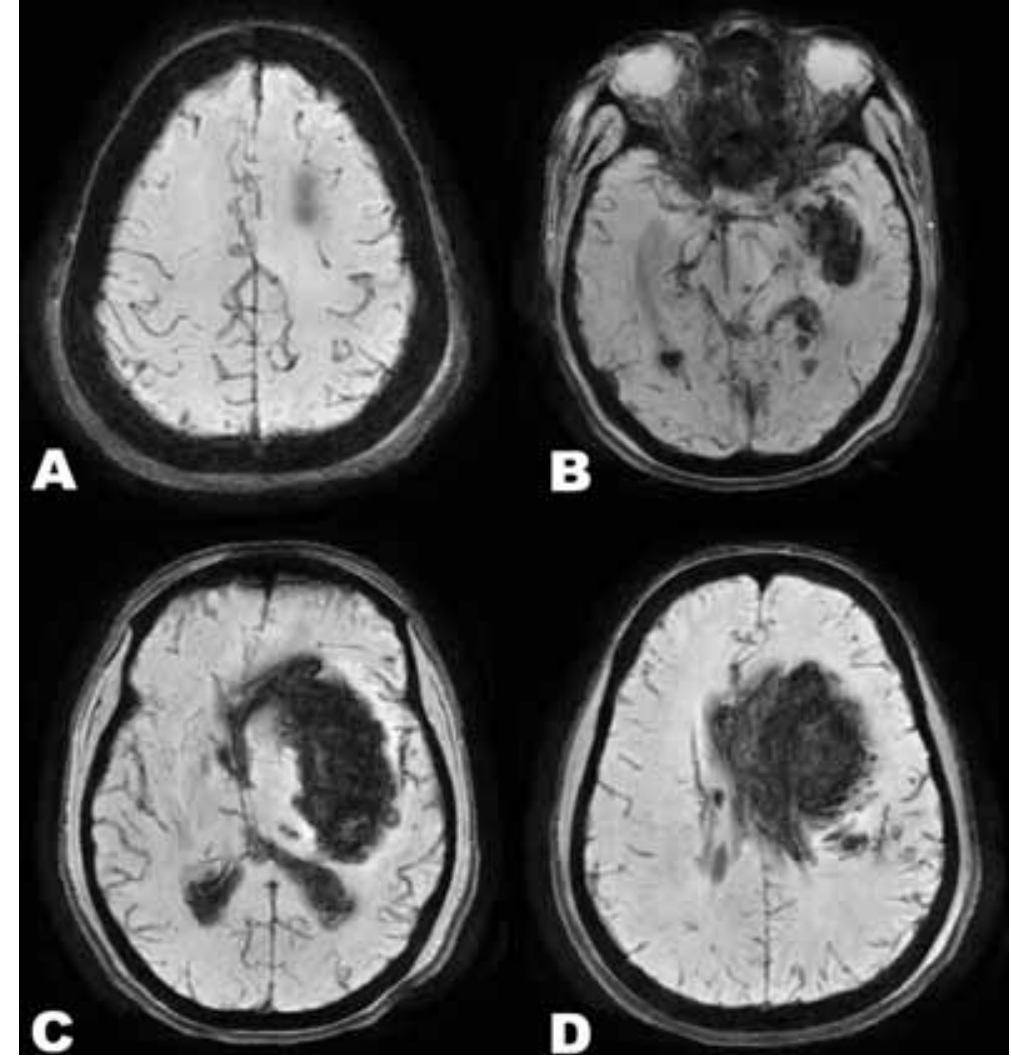

FIGURE 7. Susceptibility weighted images (A) showing prominent veins due increased susceptibility effects of deoxyhemoglobin, (B) showing ventricular hemorrhage, left uncal herniation, microhemorrhage in midbrain? duret hemorrhage, ( $C$ and $D)$ showing parenchymal hematoma which was cause of the death in eclamptic patient.

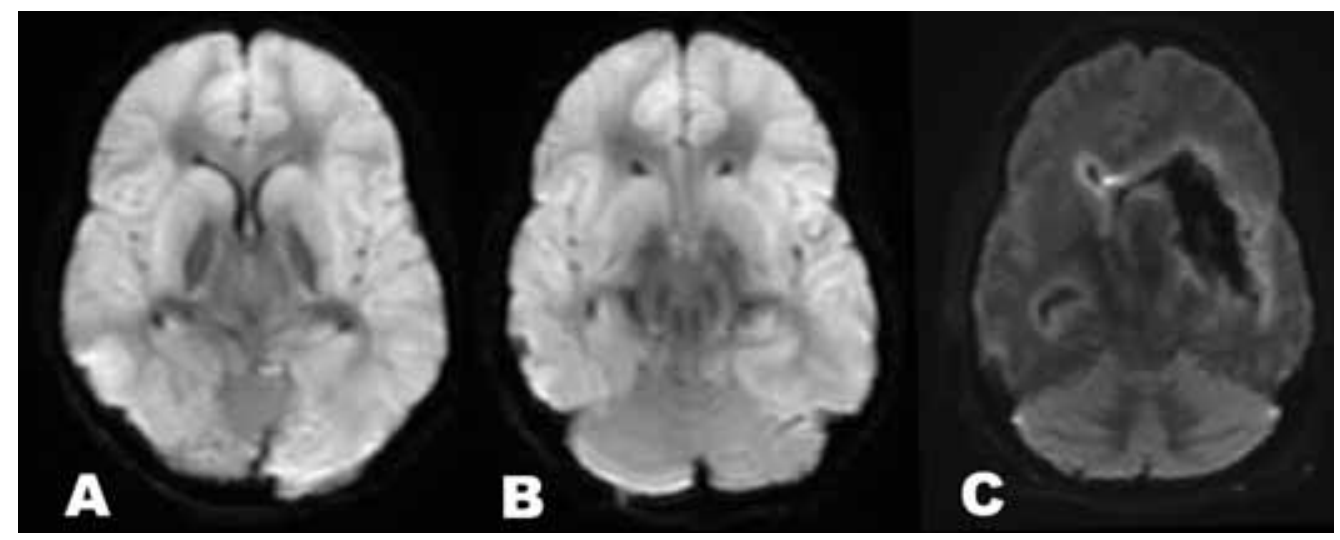

FIGURE 8. Diffusion weighted images $(A)$ and $(B)$ showing diffuse cortical restriction, image $(C)$ showing diffusion restriction in cerebellum.

specific in diagnosing brain death, however when we see a constellation of findings in a pattern they give very valuable information in diagnosing brain death. $(1,2)$

\section{CONCLUSION}

It's increasingly becoming important to diagnose brain death to avoid prolonged unnecessary futile ventilator support to patient and in the context of organ transplant. Though diagnosis of brain death is mostly clinical in most of the countries, in cases where bedside clinical tests cannot be performed due to various reasons, ancillary diagnostic tests are sought. Though contrast angiographies and radio nucleotide imaging are the mainstay in establishing the diagnosis, they are cumbersome to perform. $(2,6)$ MRI can be used as an alternate investigation for establishing brain death as it has the ability to obtain angiography without giving any contrast and gives valuable brain parenchymal detail which sometimes helps in diagnosing the cause 
of death. No single sign in MRI is specific for diagnosing brain death, so a combination of findings like loss of intracranial flow void in bilateral carotids and vertebral arteries, diffuse cerebral, cerebel-

\section{REFERENCES}

1. Lee D.H., Nathanson J.A., Fox A.J., Pelz D.M., Lownie S.P. Magnetic resonance imaging of brain death. Canadian Association of Radiologists Journal l'Association canadienne des radiologistes 1995; 46:174-178.

2. Sohn C.H., Lee H.P., Park J.B., et al. Imaging findings of brain death on 3-tesla MRI. Korean Journal of Radiology 2012;13:541-549.

3. Ali I., Wani W.A., Saleem K. Cancer scenario in India with future perspectives. Cancer Therapy 2011; 8:56-70.

4. Lövblad K.O., Basssetti C. Diffusion-weighted magnetic resonance imaging in brain death. Stroke 2000; 31:539-542. lar and brainstem swelling, uncal, tonsillar herniations can help confirm a case of clinically proven brain death. Higher Tesla MRI can minimise the scan times in acutely ill patients.
5. Matsumura A., Meguro K., Tsurushima H., et al. Magnetic resonance imaging of brain death. Neurologia medico-chirurgica 1996; 36:166-171.

6. Laurin N.R., Driedger A.A., Hurwitz G.A., et al. Cerebral perfusion imaging with technetium-99m HM-PAO in brain death and severe central nervous system injury. Journal of nuclear medicine: official publication, Society of Nuclear Medicine 1989; 30:1627-1635. 